\title{
DISTRIBUIÇÃo DE LARVAS DE Katsuwonus pelamis (PISCES, SCOMBRIDAE), EM LARGA ESCALA, NA ZONA ECONÔMICA EXCLUSIVA DO NORDESTE DO BRASIL.
}

\author{
Nilton CÉsAR TOSTA PINTO ${ }^{1}$ \\ PAULO MAFALDA JR. ${ }^{1}$ \\ CARMEM MEDEIROS ${ }^{2}$ \\ GILSON MOURA ${ }^{2}$ \\ CHRISTIANE SAMPAIO DE SOUZA ${ }^{1}$
}

${ }^{1}$ Laboratório de Plâncton, Instituto de Biologia

Universidade Federal da Bahia

Salvador, Brazil 40.210-020

${ }^{2}$ Departamento de Oceanografia

Universidade Federal do Pernambuco

Recide, Brazil 50.730-540

\begin{abstract}
RESUMO
O objetivo deste estudo foi delinear a distribuição espacial e sazonal e analisar a influência de fatores oceanográficos (temperatura, salinidade, biomassa primária e biomassa secundária) sobre a densidade de larvas de Katsuwonus pelamis. Os cruzeiros foram realizados à bordo do Navio Oceanográfico Antares da Marinha do Brasil, na Zona Econômica Exclusiva (ZEE) do Nordeste do Brasil, em 1995 (REVIZEE Nordeste I), 1997 (REVIZEE Nordeste II) e 1998 (REVIZEE Nordeste III). Realizou-se a coleta do macrozooplâncton através de arrastos oblíquos com rede bongo, com malha $500 \mu \mathrm{m}$. O bonito-barriga-listrada destacou-se como a espécie mais abundante da família Scombridae, atingindo cerca de $50 \%$ da abundância relativa total. A distribuição espacial de $K$. pelamis, em larga escala, ocorreu de forma independente em relação aos parâmetros oceanográficos estudados. Não foi registrada influência da sazonalidade uma vez que não foram detectadas diferenças significativas entre as três épocas investigadas.
\end{abstract}

Palavras chave: Ictioplâncton, Katsuwonus pelamis,Zona Econômica Exclusiva

\section{Abstract \\ Large scale distribution of Katsuwonus pelamis larvae (Pisces, Scombridae), in the Exclusive Economic Zone off North-East Brazil}

The objective was to delineate the space and seasonal distribution and to analyze the influence of oceanographic factors (temperature, salinity, primary and secondary biomass) on the density larvae of skipjack Katsuwonus pelamis. The cruises were accomplished on board of the Oceanographic Vessel "Antares" of the Brazilian Navy, in the northeast Brazilian Exclusive Economic Zone, (EEZ) during 1995 (REVIZEE Nordeste I), 1997 (REVIZEE Nordeste II) and 1998 (REVIZEE Nordeste III). The sampling of the macrozooplankton was carried out by oblique tows using a bongo net, mesh size of $500 \mu \mathrm{m}$. Katsuwonus pelamis stood out as the most abundant species among Scombridae, reaching about $50 \%$ of the total relative abundance. The large scale distribution of $K$. pelamis larvae occurred in an independent way in relation to the physical and 
chemical parameters studied. Significant differences were not detected among the oceanographic cruises (ANOVA, p>0.05). This suggests that its presence probably has no direct relationship with seasonality.

Key words: Ichthyoplankton, Katsuwonus pelamis,Exclusive Economic Zone

\section{INTRODUÇÃO}

A Zona Econômica Exclusiva nordestina se encontra em uma região oceânica tropical, constituída por um sistema oligotrófico, que possui uma teia alimentar complexa, porém com baixa produtividade biológica (Ekau \& Knoppers,1999). Quando as águas da Corrente Sul Equatorial (CSE), atingem a plataforma continental do Nordeste, entre $11^{\circ} \mathrm{S}$ e $15^{\circ} \mathrm{S}$, elas se bifurcam e um ramo segue para o norte, originando a Corrente Norte do Brasil (CNB), enquanto o outro segue para o sul, formando a Corrente do Brasil (CB) (Peterson \& Stramma,1991). Os baixos níveis de nutrientes, biomassa fitoplanctônica e biomassa zooplanctônica (Becker,2001; Medeiros et al.,1999; Neumann-Leitão et al.,1999) são compensados na zona oceânica, por ressurgências topográficas, causadas por ilhas e montes submarinos (Ekau et al.,1999; Travassos et al.,1999). Nos bancos da Cadeia Norte Brasileira, uma pronunciada ressurgência, em forma de domo, ocorre como conseqüência da interação entre correntes oceânicas e o relevo submarino (Travassos et al.,1999). Na zona costeira a exportação do material de manguezais, via descarga fluvial, e a ressurgência de borda de plataforma são mecanismos que contribuem para a produtividade da águas do Nordeste (Medeiros et al,1999).

No início da década de 90 os levantamentos sobre ictioplâncton eram considerados raros no nordeste do Brasil (Brandini et al.,1997). Contudo, em meados da década de 90 os estudos sobre ictioplâncton passaram a ser realizados de forma mais freqüente na região costeira e oceânica (Carrozzo et al.,1999; Ekau et al.,1999; Lessa et al.,1999; Mafalda Jr. \& Silva,1996; Mafalda Jr. et al.,1998; Mafalda Jr. et al.,1999; Mafalda Jr.,2000; Nascimento,2000; Silva,1997; Souza,1999; Velame,2001). A ocorrência de larvas de Scombridae no ictioplâncton do mar nordestino, foi registrada por: Matsuura (1984) que estudou a abundância de K. pelamis nos Arquipélagos de Fernando de Noronha e de São Pedro e São Paulo. Mafalda Jr. (2000) que observou a ocorrência de larvas de Scomberomorus cavalla e Auxis thazard, na costa norte da Bahia e Nascimento (2000) que encontrou larvas de K. pelamis e Thunus no ictionêuston da ZEE Nordeste. Em águas tropicais os estudos sobre distribuição e abundância de larvas de $K$. pelamis vem sendo desenvolvidos, principalmente no Oceano Pacífico (Leis et al.,1991; Boehlert \& Mundy,1994).

Os peixes da família Scombridae, que incluem os atuns e bonitos, distribuem-se em mares tropicais e subtropicais, entre os paralelos $35{ }^{0} \mathrm{~N}$ e $35{ }^{0} \mathrm{~S}$, onde constituem importantes recursos pesqueiros (Nishikawa \& Rimmer, 1987). No oceano Atlântico várias espécies de atuns já se encontram com seus estoques sobrexplotados (ICCAT,1994). No Brasil, o bonito-barriga-listrada, K. pelamis, é capturado pela frota industrial apenas em frente à costa das regiões sul e sudeste (Paiva,1997). Na região nordeste outras espécies de Scombridae são capturados comercialmente na Cadeia Norte Brasileira, Arq. de Fernando de Noronha e Arq. de São Pedro e São Paulo (Hazin,1994).

Através do Programa de Avaliação do Potencial Sustentável de Recursos Vivos na Zona Econômica Exclusiva (REVIZEE), foram realizados levantamentos de dados oceanográficos e amostragens de ictioplâncton, entre 1995 e 1998 (Carrozo et al.,1999). Estes dados foram utilizados para descrever a distribuição espacial e sazonal da família Scombridae, com ênfase no bonitobarriga-listrada, Katsuwonus pelamis (Linnaeus), além de analisar a influência de fatores oceanográficos (temperatura, salinidade, clorofila e biovolume de macroplâncton) sobre a 
abundância de suas larvas, na ZEE Nordeste. Este trabalho também foi desenvolvido com o objetivo de testar as hipóteses de que a distribuição em larga escala e a abundância de larvas de Katsuwonus pelamis são influenciadas pela sazonalidade.

\section{Metodologia}

As amostras foram coletadas a bordo do Navio Oceanográfico Antares, da Marinha do Brasil, durante as Campanhas REVIZEE Nordeste I, realizada entre 02 de Agosto e 26 de Outubro de 1995; REVIZEE Nordeste II, que ocorreu entre 21 de Janeiro e 13 de Abril de 1997 e REVIZEE Nordeste III, realizado entre 28 de Abril e 20 de Julho de 1998. Neste período foram percorridas 441 estações oceanográficas, localizadas entre 12 e 200 milhas náuticas da costa (Fig. 1). Em função da grande extensão da grade amostral não foi definida uma situação climática única, para cada campanha do REVIZEE, que por isto foram referenciadas apenas como: Nordeste I, II e III. Em todas as campanhas foram amostradas as áreas: Arquipélago de São Pedro e São Paulo, Arquipélago de Fernando de Noronha, Cadeia Norte Brasileira, Área Oceânica Norte, Área Oceânica Leste, Área de Plataforma Norte (Piauí - Rio Grande do Norte) e Área de Plataforma Leste (Rio Grande do Norte - Bahia).

A caracterização das massas de água foi realizada através de registros de salinidade, em UPS e de temperatura, em graus Celsius $\left({ }^{\circ} \mathrm{C}\right.$ ), obtidos através de perfilagem contínua (CTD), ao longo da coluna d’água. As amostras de água destinadas às análises de biomassa primária fitoplanctônica, foram obtidas por meio de garrafas de Niskin, em sistema de Roseta. Após a filtração das amostras à bordo, os filtros foram congelados para posterior análise de clorofila a, através do método espectrofotométrico de Strickland \& Parsons (1972). A biomassa fitoplanctônica, expressa em $\mu \mathrm{g} / \mathrm{l}$, foi obtida no nível de 50 \% de intensidade luminosa. O ictioplâncton foi coletado segundo metodologia proposta por Smith \& Richardson (1977), através de rede do tipo Bongo, com abertura de $50 \mathrm{~cm}$ e malha 500 micrômetros $(\mu \mathrm{m})$, dotada de fluxômetro, para o cálculo do volume de água filtrada.

Arrastos oblíquos, com duração média de 10 minutos, foram realizados à partir dos 5 metros de profundidade em estações rasas, e 100 metros (Nordeste I) ou 200 metros de profundidade (Nordeste II e III), nas demais estações. As amostras foram acondicionadas em frascos plásticos de $1000 \mathrm{ml}$, em solução de formol a 4 \%, neutralizado com tetraborato de sódio. No laboratório, foram submetidas a estimativas do biovolume de macroplâncton, através do processo de sedimentação durante 24 horas. Os resultados de biomassa secundária foram expressos em ml/100 $\mathrm{m}^{3}$

A triagem total de ovos, larvas e jovens de peixes, foi realizada através de microscópio estereoscópico, visando a sua identificação e quantificação. A identificação das larvas e jovens de peixes foi realizada ao nível de família. Para a identificação das larvas de Scombridae foram utilizados os trabalhos de Fahay (1983) e Moser et al. (1984). A sinopse taxonômica se baseou na classificação apresentada por Nelson (1994).

A abundância relativa (\%) foi calculada de acordo com a fórmula: $\mathrm{Ar}=(\mathrm{Na} * 100) / \mathrm{NA}$, onde, Na é o número de larvas de K. pelamis e NA é o número total de larvas de peixes. A seguir, este taxon foi classificado da seguinte forma: > $50 \%$ - dominante; 50-30 \% - abundante; 30-15\% pouco abundante; $15-5 \%$ - rara e $<5 \%$ - esporádica. A freqüência de ocorrência foi determinada pela fórmula: Fo $=\left(\mathrm{Ta}^{*} 100\right) / \mathrm{TA}$, onde Ta é o número de amostras onde K. pelamis ocorreu e TA é o número total de amostras. Este taxon foi classificado segundo a escala de Neumann - Leitão (1994): > $70 \%$ - muito freqüente; $70-40 \%$ - freqüente; $40-10 \%$ - pouco freqüente e < $10 \%$ esporádica.

Tropical Oceanography, Recife: v. 30, n. 2, p. 119-131, 2002 


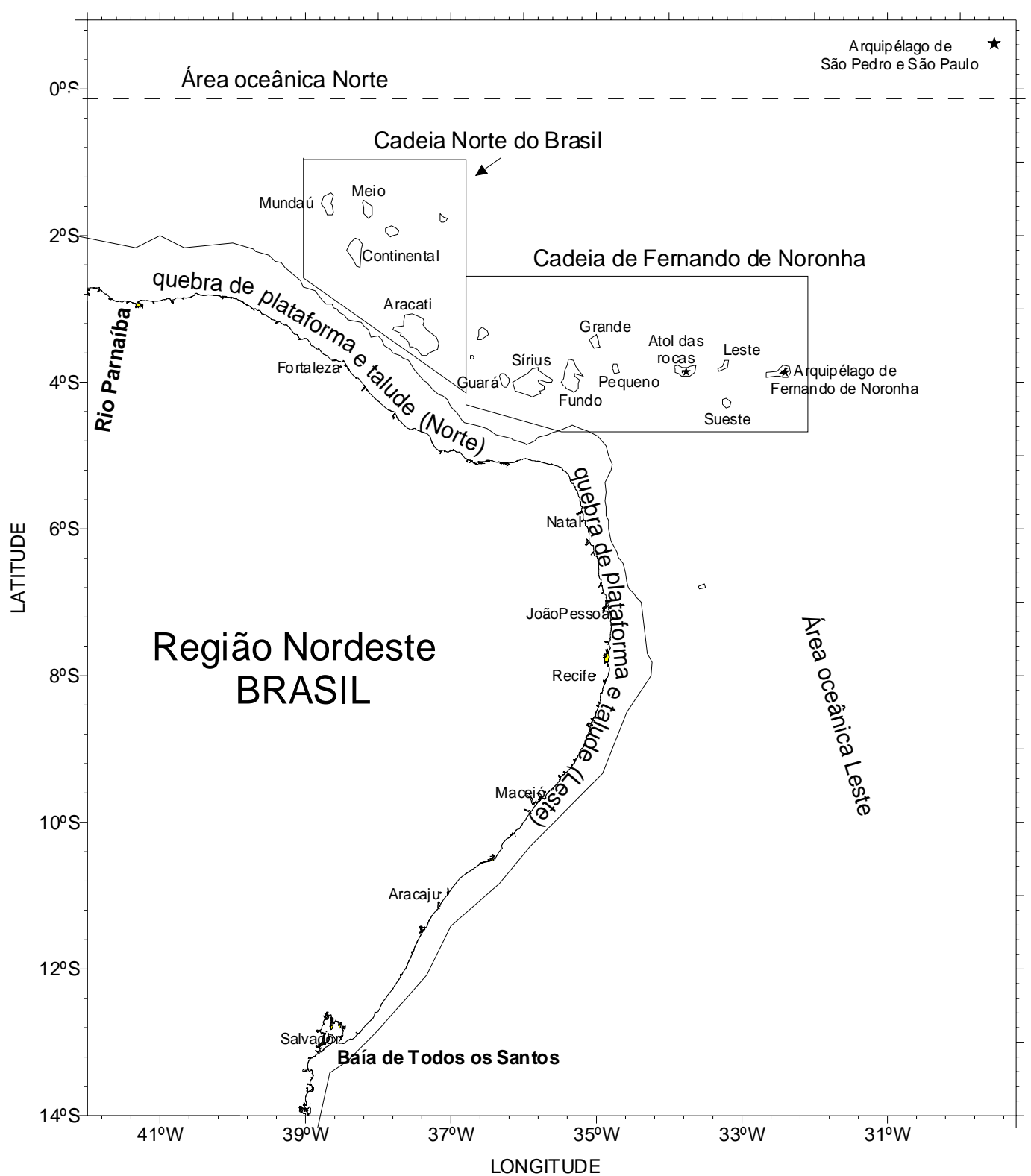

Figura 1 - Áreas de amostragem, na Zona Econômica Exclusiva, durante o REVIZEE Nordeste I, II, III.

A densidade (N/100 $\mathrm{m}^{3}$ ), foi obtida a partir do quociente entre o número total de larvas de K. pelamis obtidas em cada amostra $(\mathrm{N})$ e o volume de água filtrada pela rede de plâncton, através da fórmula: $\mathrm{N} / 100 \mathrm{~m}^{3}=(\mathrm{N} / \mathrm{V}) * 100$. A análise de variância foi empregada visando verificar a existência de diferença significativa entre as épocas investigadas. Foi aplicado o Teste de Kolmogorov - Smirnov para se avaliar a normalidade e o Teste de Bartlet para verificar a homogeneidade das variâncias. Como as variâncias não eram homogêneas, aplicou-se a ANOVA não paramétrica de Kruskal - Wallis (Zar, 1984), seguida do teste de comparações múltiplas de

Tropical Oceanography, Recife: v. 30, n. 2, p. 119-131, 2002 
Dunn. Os dados de densidade foram representados em mapas através do software SURFER for windows, versão 6 (Keeler, 1995).

\section{RESUltados}

\section{Condições Oceanográficas}

A distribuição horizontal da temperatura do mar à superfície apresentou-se bastante homogênea, com valores oscilando entre 25,49 e $27,77^{\circ} \mathrm{C}$ (média $=26,57^{\circ} \mathrm{C}$ ), durante o Nordeste I, entre 27,19 e 28,79 (média $=28,05{ }^{\circ} \mathrm{C}$ ), durante o Nordeste II e entre 25,85 e 29,49 (média = 28,05 ${ }^{\circ} \mathrm{C}$ ) durante o Nordeste III. A variação sazonal da temperatura foi constatada através da diferença significativa (ANOVA, p<0,0001), entre as campanhas, em função de uma elevação global da temperatura das águas superficiais, de cerca de $1,5^{\circ} \mathrm{C}$, encontrada no Nordeste II e III, em relação ao Nordeste I (Teste de Dunn, $\mathrm{p}<0,05)$. Em todas as épocas estudadas foi constatada a presença da massa de Água Equatorial Superficial (AES), entre a superfície e os $150 \mathrm{~m}$ de profundidade.

A salinidade nas camadas mais superficiais da Água Equatorial Superficial, oscilou entre 35,49 e 37,24 UPS (média=36,19) durante o Nordeste I, entre 34,78 e 37,22 UPS (média - 36,20), durante o Nordeste II e entre 35,54 e 37,41 UPS (média - 36,40), durante o Nordeste III. A análise variância apontou diferença significativa entre as campanhas (ANOVA, $\mathrm{p}<0,0001$ ), indicando ocorrência de variação temporal da salinidade. Posteriormente, o teste de comparações múltiplas de Dunn $(p<0,001)$, verificou que esta diferença ocorreu em função da maior salinidade encontrada no Nordeste III, em relação ao Nordeste I e II. Não foi encontrada correlação significativa entre a temperatura, a salinidade e a densidade de larvas de K. pelamis.

\section{Biomassa primária}

O Nordeste I apresentou um padrão de distribuição bastante homogêneo, com os registros de clorofila situando-se entre 0,01 e $1 \mu \mathrm{g} / \mathrm{l}$, em toda ZEE Nordeste. No Nordeste II, apesar da maioria dos resultados estarem situados na faixa de 0,01 e $1 \mu \mathrm{g} / \mathrm{l}$, valores entre 1 e $4 \mu \mathrm{g} / \mathrm{l}$, foram registrados próximo à desembocadura do rio Parnaíba, entre a plataforma continental de Alagoas e Sergipe, na plataforma continental do norte da Bahia, além da área oceânica norte. No Nordeste III as estações de amostragem com valores situados entre 1 e $4 \mu \mathrm{g} / \mathrm{l}$, distribuíram-se em toda a área de plataforma continental norte e leste. Na área oceânica se destacaram a Cadeia Norte Brasileira, o Arquipélago de Fernando de Noronha, além da área oceânica norte. O teste de Kruskal-Wallis indicou diferença significativa ( $<<0,0001)$, nos valores de biomassa primária durante as épocas investigadas. O teste de Dunn ( $p<0,001)$, verificou que no Nordeste I foram obtidas as concentrações mais reduzidas de clorofila a, com valores intermediários no Nordeste II e mais elevados no Nordeste III. Porém, não foi encontrada correlação significativa entre a biomassa primária e a densidade de larvas de K. pelamis.

\section{Biomassa secundária}

Durante o Nordeste I, valores entre 50 e $200 \mathrm{ml} / 100 \mathrm{~m}^{3}$, foram encontrados apenas em áreas oceânicas da Cadeia Norte Brasileira e Arq. de Fernando de Noronha, enquanto que, no Nordeste II, também estiveram localizados entre a plataforma continental sul (entre Alagoas e norte da Bahia) e no Nordeste III entre a plataforma continental norte (entre Rio Grande do Norte e Piauí). O teste de Kruskal-Wallis indicou diferença significativa $(\mathrm{p}<0,0001)$ nos valores de 
biomassa secundária entre as épocas de estudo. O teste de Dunn $(\mathrm{p}<0,001)$, verificou que no Nordeste I foram obtidas as concentrações mais reduzidas de macroplâncton, com valores intermediários no Nordeste II e mais elevados no Nordeste III. Contudo, não foi encontrada correlação significativa entre a biomassa secundária e a densidade de larvas de K. pelamis.

\section{Distribuição e abundância de larvas de peixes}

Durante o Nordeste I, os valores situados entre 50 - 500 larvas $/ 100 \mathrm{~m}^{3}$, estiveram localizados na Cadeia Norte Brasileira e Arq. de Fernando de Noronha, enquanto que, no Nordeste II, também foram encontrados na plataforma continental sul (entre Alagoas e norte da Bahia). No Nordeste III os maiores valores foram encontrados na plataforma continental sul (entre Paraíba e Bahia) e plataforma continental norte (entre Ceará e Piauí). O teste não-paramétrico de KruskalWallis indicou diferença significativa $(\mathrm{p}<0,0001)$ na densidade de larvas de peixes. $\mathrm{O}$ teste de comparações múltiplas de Dunn $(\mathrm{p}<0,001)$ constatou uma diferença entre o Nordeste I, com relação ao Nordeste II e Nordeste III.

Os resultados obtidos para as densidades de larvas de peixes refletem aqueles encontrados para biomassa primária e biomassa secundária, indicando que a influência da sazonalidade térmica colaborou para a ocorrência de valores mais elevados no Nordeste II e no Nordeste III, em relação ao Nordeste I.

\section{Distribuição e abundância de larvas de Scombridae e de Katsuwonus pelamis}

A família Scombridae esteve constituída pelos seguintes taxa, com suas respectivas abundâncias relativas: Katsuwonus pelamis (56,5 \%), Thunnus sp (39,1 \%), Acantocybium solandri (2,2 \%), Euthynnus aletteratus (1,1\%), Scomberomorus sp (1,1\%).

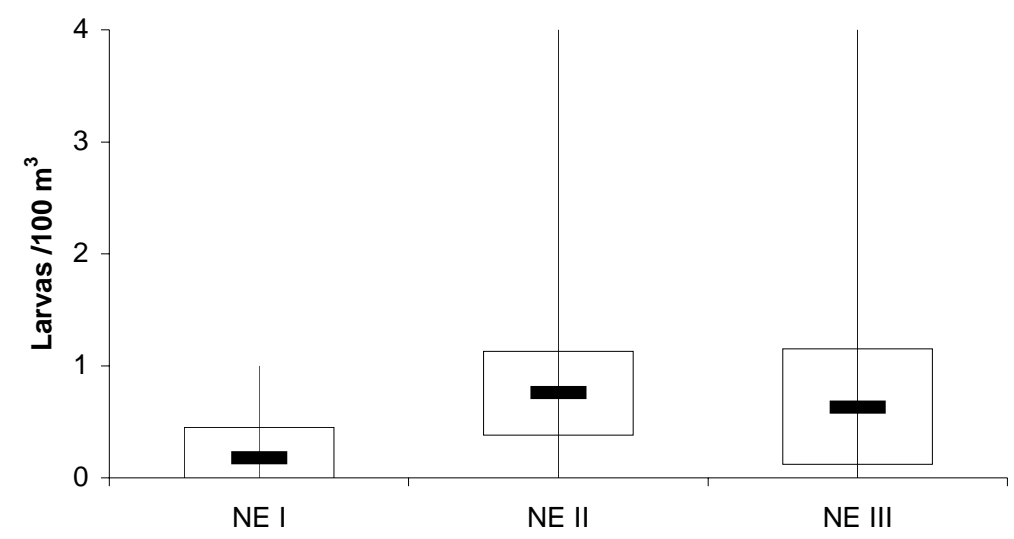

Figura 2 - Densidade média de Katsuwonus, pelamis $/ 100 \mathrm{~m}^{3}$, obtida durante o REVIZEE Nordeste I, Nordeste II e Nordeste III (valores máximos e mínimos: linha vertical; média: barra horizontal; intervalo de confiança: retângulo). 
Entre os estágios larvais de K.pelamis identificados predominam as pós-larvas medindo entre 4,5 e 8,0 mm. Não foram encontrados estágios recém-eclodidos e jovens de K.pelamis. A densidade média de larvas de K. pelamis (Fig. 2), oscilou entre 0,76 larvas $/ 100 \mathrm{~m}^{3}$, no Nordeste II, seguida de 0,63 larvas $/ 100 \mathrm{~m}^{3}$, no Nordeste III, até apenas 0,18 larvas $/ 100 \mathrm{~m}^{3}$, no Nordeste I. Contudo, em função das baixas densidades encontradas nas três épocas investigadas, a ANOVA de Kruskal-Wallis não encontrou diferença significativa ( $p>0,05)$, entre as campanhas.

No Nordeste I (Fig. 3) as larvas do bonito foram encontradas apenas no Arq. de Fernando de Noronha (AFN), Área Oceânica Leste (AOL), além da Área de Plataforma Sul (entre Alagoas e norte da Bahia).

As larvas de K. pelamis foram consideradas raras, pois contribuíram com até 2,7 \%, do total de larvas de peixes. Durante o Nordeste II $K$. pelamis ampliou a sua área anterior de distribuição ocorrendo também na Cadeia Norte Brasileira (CNB), e nas áreas oceânicas norte e sul (Fig. 3). Porém, novamente as larvas de $K$. pelamis foram consideraras raras por contribuírem com abundância relativa máxima de 2,3 \%. Ao longo do Nordeste III, K. pelamis foi capturado no Arq. de Fernando de Noronha, na Cadeia Norte Brasileira e nas áreas oceânicas norte e leste (Fig. 3). Nesta campanha as larvas de K. pelamis contribuiram com um máximo de 0,9 \% do total de larvas de peixes. As larvas de bonito não foram encontradas na plataforma continental norte e no Arquipélago de São Pedro e São Paulo.

Apesar da pequena contribuição de K. pelamis no total de larvas de peixes, sua importância é relevante no contexto da família Scombridae, uma vez que mais de $50 \%$ das larvas pertencem a esta espécie. No Nordeste I, K. pelamis atingiu 56,3 \% da abundância relativa; durante o Nordeste II foram 59,2 \% e no Nordeste III atingiram 53,9 \% do total de larvas de Scombridae. A freqüência de ocorrência de $K$. pelamis foi de 5,3 \%, no Nordeste I, 6,9 \% no Nordeste II e, 12,9 \% no Nordeste III. 

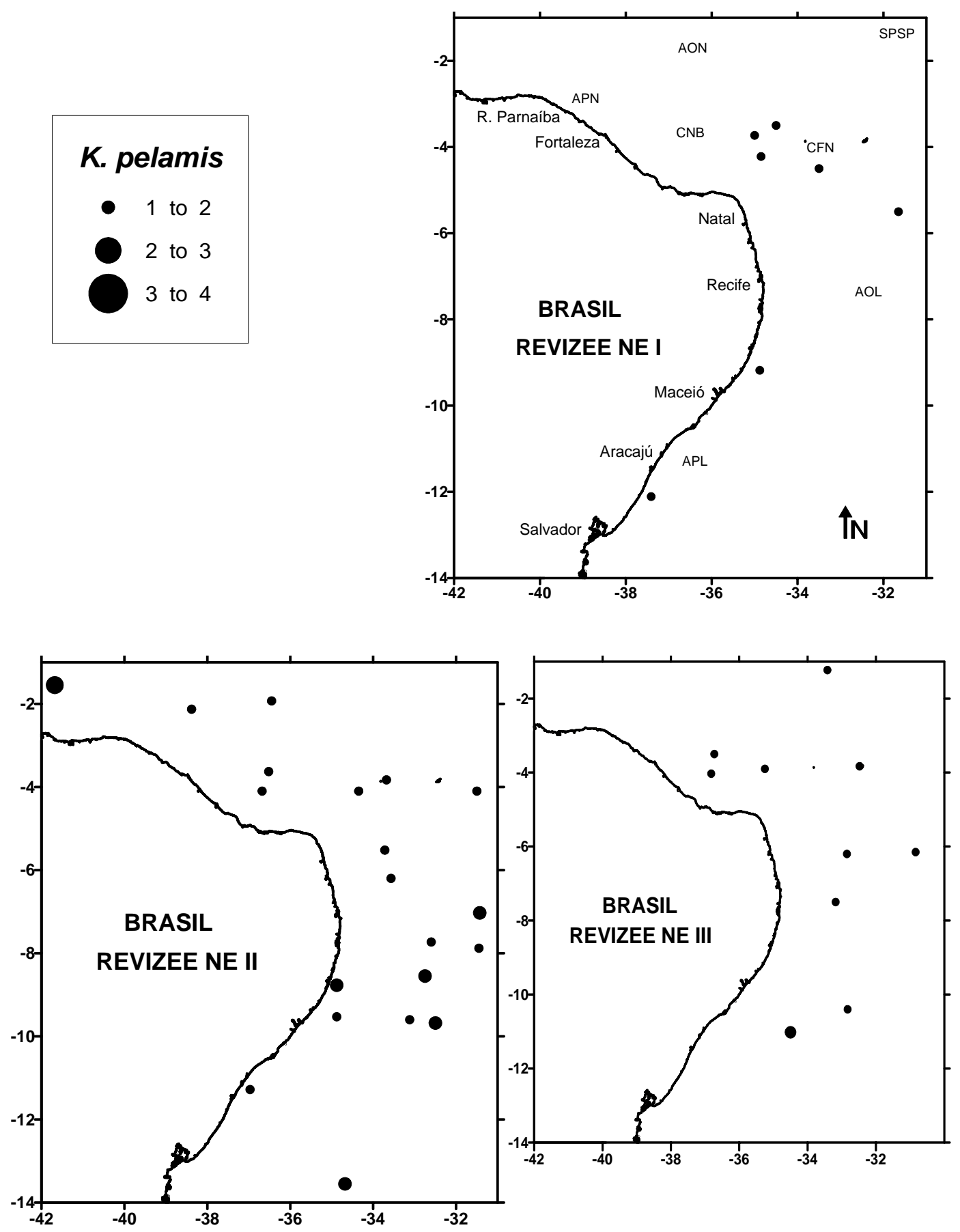

Figura 3 - Distribuição espacial das larvas de katsuwonus pelamis / 100m³ , durante o REVIZEE Nordeste I, Nordeste II e Nordeste III. (APL: Área de Plataforma Leste; APN: Área de Plataforma Norte; AOL: Área Oceânica Leste; AON: Área Oceânica Norte; AFN: Arquipélago de Fernando de Noronha; CNB: Cadeia Norte Brasileira; SPSP: Arquipélago de São Pedro e SDIBuiless)são 
As coletas de ictioplâncton foram realizadas entre a superfície e uma profundidade máxima de 200 metros, conduzindo a amostragem das larvas de peixes que se encontravam em uma das três massas de água características da ZEE Nordeste: Água Equatorial Superficial (AES), com temperatura superior à $26{ }^{\circ} \mathrm{C}$, e salinidade entre 35,5 e 36,5 UPS, ocupando uma faixa entre a superfície e os $200 \mathrm{~m}$; Água de Máxima Salinidade Subtropical (AMS), com temperatura entre 20 ${ }^{\circ} \mathrm{C}$ e $26{ }^{\circ} \mathrm{C}$ e salinidade entre 36 e 37 UPS, localizada acima do topo da termoclina, entre 50 e 200 $\mathrm{m}$ e, a Água central do Atlântico Sul (ACAS), com temperatura entre $5{ }^{\circ} \mathrm{C}$ e $20{ }^{\circ} \mathrm{C}$ e salinidade entre 34,3 e 36 UPS (Medeiros et al.,1999 e Becker,2001). Contudo, como a desova de K. pelamis ocorre em mares tropicais, com temperatura da água da superfície à $24{ }^{0} \mathrm{C}$ (Matsuura \& Sato,1981), e suas larvas normalmente ocorrem acima da termoclina, entre 20 e $60 \mathrm{~m}$ de profundidade (Richards,1971), podemos inferir que as larvas de K. pelamis estiveram associadas principalmente a AES.

A sazonalidade térmica provocou menores temperaturas no Nordeste I e temperaturas mais elevadas no Nordeste II e III, que aliada a maior disponibilidade de nutrientes nitrogenados e fosforados (Becker,2001), colaborou para a geração de um gradiente de biomassa primária, biomassa secundária e densidade de larvas de peixes, com menores concentrações no Nordeste I, em relação ao Nordeste II e III, o que foi comprovado estatísticamente.

Durante todas as campanhas estudadas, sítios de crescimento de larvas de peixes e de larvas de K. pelamis, foram localizados principalmente na Cadeia Norte Brasileira e no Arquipélago de Fernando de Noronha, onde a ocorrência de ressurgências topográficas, causadas por ilhas e montes submarinos, promove um aumento na concentração de nutrientes inorgânicos. A formação de zonas de criação de K. pelamis também foi registrada nas Ilhas oceânicas da Polinésia Francesa (Leis et al.,1991). As larvas de K. pelamis também foram encontradas em zonas costeiras do norte da Bahia e norte de Alagoas, onde as descargas fluviais e a ressurgência de bordo de plataforma contribuem para o aumento da produtividade da águas do nordeste. Os sítios de desova de peixes também estiveram localizados ao longo de toda a plataforma continental do nordeste, mas também na Cadeia Norte Brasileira e no Arq. de Fernando de Noronha (Carrozo et al.,1999).

Os valores mais elevados de fitoplâncton, zooplâncton e ictioplâncton, encontrados em determinadas áreas costeiras e oceânicas do Nordeste, podem ser atribuídos à contribuição de estuários e manguezais, ressurgência de borda de plataforma, além da ressurgência topográfica, que podem afetar as condições oceanográficas, ao provocarem um aumento da concentração de nutrientes, e consequentemente da biomassa primária, biomassa secundária, densidades de ovos e larvas de peixes e do próprio potencial pesqueiro da região (Becker, 2001; Carrozo et al.,1999; Ekau et al.,1999; Hazin,1994; Medeiros et al.,1999; Travassos et al.,1999).

Katsuwonus pelamis é uma das espécies de bonitos mais abundantes da nossa costa, representando o mais importante recurso pesqueiro da família Scombridae (Paiva, 1997). Apesar de contribuírem com mais da metade da abundância relativa da família Scombridae, as larvas de $K$. pelamis apresentaram baixa freqüência de ocorrência e densidade, ocupando um máximo de 2,7 \% do total de larvas de peixes capturadas. Na costa leste do Brasil, Matsuura (1982), encontrou abundância relativa que atingiu 10,4 \% do total de larvas capturadas. Mafalda Jr. (1989) encontrou abundância relativa de 3,3 \% do total de larvas de teleósteos, na plataforma continental do Rio Grande do Sul. Ao longo da plataforma continental Brasileira, as densidades mais elevadas de $K$. pelamis foram encontradas no Banco de Abrolhos, na Bahia (Matsuura,1984). Nonaka et al. (2000) consideram o banco de Abrolhos a principal área de ocorrência de família Scombridae na região central do Brasil.

Em menor escala fatores ambientais como: correntes marinhas, temperatura, biomassa primária e secundária, além da ocorrência de predadores, afetam a dispersão do ictioplâncton (Doyle et al,1993; McGowen1993). Contudo, em larga escala a abundância deve estar relacionada com a

Tropical Oceanography, Recife: v. 30, n. 2, p. 119-131, 2002 
distribuição das populações dos peixes adultos e suas estratégias de desova, que são influenciadas pelos padrões oceanográficos dominantes (Norcross \& Shaw,1984; Moser \& Smith,1993). Na zona econômica exclusiva do nordeste, a ocorrência de pós-larvas de K. pelamis, nas áreas sob influência da Corrente Norte do Brasil e da Corrente do Brasil, indica a presença de populações adultas desovando em ambos sistemas de circulação, que se originam a partir da Corrente Sul Equatorial (Petterson \& Stramma,1991) e que imprimem o padrão oceanográfico dominante.

Na zona econômica exclusiva do Nordeste do Brasil. a ocorrência de larvas de K. pelamis pode estar mais relacionada com a estratégia de desova anual empregada pelos adultos, do que com a sazonalidade térmica No Hawaí a distribuição em pequena escala das larvas de K. pelamis indica maiores abundancias nas águas com temperaturas mais elevadas (Boehleet \& Mundy,1994). Matsuura (1984) e Nascimento (2000), abordando a distribuição das larvas de K. pelamis, no ictioplâncton e ictioneuston, respectivamente, sugerem que a desova da população desta espécie ocorra durante todo o ano na ZEE Nordeste. Na costa leste do Brasil sua desova ocorre na primavera e durante o verão (Matsuura,1982; Zavala-Camin,1978), enquanto que, na costa sul do Brasil, ocorre apenas no verão (Mafalda Jr.,1989), assim como na África do Sul (Beckley \& Leis,2000).

A distribuição espacial em larga escala, das larvas de K. pelamis na ZEE NORDESTE, ocorreu sem uma relação direta com a sazonalidade, uma vez que não foram encontradas diferenças significativas na concentração de suas larvas, ao longo das campanhas oceanográficas. Porém, em regiões tropicais do Oceano Pacífico, já foi demonstrado que a estrutura física da coluna de água, particularmente a temperatura e a salinidade, alteram os padrões de distribuição espacial e sazonal das larvas de K. pelamis, porém em pequena escala (Boehleet \& Mundy,1994).

\section{AgRAdecimentos}

Os autores agradecem ao Ministério do Meio Ambiente e da Amazônia Legal (MMA) e à Comissão Interministerial dos Recursos do Mar (CIRM), pelo financiamento do Programa REVIZEE. Ao CNPq pelas bolsas de Desenvolvimento Tecnológico Industrial e pela bolsa de Mestrado em Ecologia e Biomonitoramento. As equipes da área de Oceanografia que atuaram na coleta de dados, à bordo do N/Oc ANTARES, durante as Comissões Oceanográficas REVIZEE Nordeste I, II e III.

\section{REFERÊNCIA BIBLIOGRÁFICA}

BECKER M. Hidrologia dos Bancos e Ilhas oceânicas do Nordeste Brasileiro, uma

contribuição ao Programa REVIZEE. São Carlos, 2001. 151.Universidade Federal de São Carlos. Centro de Ciências Biológicas e da Saúde, Programa de Pós - Graduação em Ecologia e Recursos Naturais.

BECKLEY LE, LEIS J. M. Occurrence of tuna and mackerel larvae (Family Scombridae) off the east coast of South Africa. Marine and Freshwater Research. v. 51. n. 8, p. 777-782. 2000.

BOEHELERT, G. W.; MUNDY, B. C. Vertical and onshore-offshore distributional patterns of tuna larvae in relation to physical habitat features. Mar. Ecol. Progress. Series, v. 107, n. 1-2, p. 1-13. 1994.

BRANDINI, F. P.; LOPES, R. M.; GUTSEIT, K. S.; SPACH, H. L.; SASSI, R. Planctonologia na plataforma continental do Brasil. REVIZEE. MMA, CIRM, FEMAR, 196p. 1997. 
CARROZZO G.; MAFALDA Jr,. P.; PINTO, N. C. T.; LIMA, D. L. L.; VELAME, M. P. B. Distribuição espacial e temporal do ictioplâncton ao longo da zona econômica exclusiva nordestina (ZEE-NE), entre Salvador, Bahia e Rio Parnaíba, Piauí. In: CONGRESSO LATINOAMERICANO SOBRE CIÊNCIAS DEL MAR, 18, Trujillo, Anais ... 1999.

DOYLE, M. J.; MORSE, W. W. ; KENDALL Jr., A. W. A comparision of larval fish assemblages in the temperate zone of northeast Pacific and the northwest Atlantic ocean. Bull. Mar. Sci., v. 53, p. 588-644,. 1993.

EKAU, W.; KNOPPERS, B. An introduction to the pelagic system of the north-east and east Brazilian shelf. Archive of Fishery and Marine Research, v. 47, n. 2-3, p. 113-132. 1999.

EKAU, W.; WESTHAUS-EKAU, P.; MEDEIROS, C. Large scale distribution of fish larvae in the continental shelf waters off north-east Brazil. Archive of Fishery and Marine Research. v. 47, n. 2-3), p. 183-200. 1999.

FAHAY, M. Guide to the early stages of marine fishes. Journ.North.Atl.Fish.Soc., 4. 1983, 423p.

HAZIN, F. H. V. Fisheries-oceanographical study on tunas, billfishes and sharks in the southwestern equatorial Atlantic ocean. 1994.286p. D.Sc. These. Tokyo University of Fisheries. Department of Marine Science and Tecnology.

ICCAT Report for biennial period, 1994-1995. Part I. International Commission for the Conservation of Atlantic Tunas, Madrid, 1994.283p.

KEELER, D. SURFER for Windows. Version 6. User`s Guide. 1995.

LEIS, J. M;. TRNSKI, T.; HRMELINVIVIEN, M.; RENON, J. P.; DUFOUR, V. ELMOUDNI, M. K.; GALZIN, R. High concentrations of tuna larvae (Pisces, Scombridae) in near reef waters of French-Polynesia (Society and Tomuatu islands). Bull. Mar. Sci., 48(1):150-158. 1991.

LESSA, R. P. LUCCHESI, R. B.; MAFALDA Jr., P,; VASKE Jr., T.; BEZERRA Jr., J. L.; ROPKE, A. Ecological aspects of neuston fish larvae, around islands, rocks and oceanic banks in northern Brazilian coast (Leg 4 - Jops II). In; COLACMAR, 8, Santos, Resumos expandidos. IOUSP/ALICMAR/FIESP, 2:76-77. 1997.

LESSA, R. P.; MAFALDA Jr., P.; ADVÍNCULA, R.; LUCCHESI, R.; BEZERRA Jr., J. L.; VASKE Jr., T.; HELLEBRANDT, D. Distribution and abundance of ichthyoneuston at seamounts and islands off north-eastern Brazil. Archive of Fishery and Marine Research, v. 47, n.2/3, p.:133-146. 1999.

MAFALDA, Jr. P. Identificação, ocorrência e abundância de larvas da subordem Scombroidei (Pisces - Peciformes) no Atlântico Sul Ocidental (25 - 40 S). Rio Grande, 1989, 76f. Dissertação (Mestrado), FURG.

MAFALDA Jr., P.; SILVA, V. R. F. Caracterização do ictioplâncton do sistema estuarino-lagunar de Jequiá, Alagoas. Bol. Estud. Ciênc. Mar. v. 9, p. 89-104. 1996 
MAFALDA Jr., P.; LESSA, R. P.; KLOPFER, M. VELAME, M. P. Abundância de larvas de Pleuronectiformes nos bancos oceânicos da Cadeia Norte Brasileira, durante o verão de 1995. In: SEMANA NACIONAL DE OCEANOGRAFIA, 10, Itajaí. Anais... p.119-121. 1997.

MAFALDA Jr. P.; SINQUE, C.; CARROZZO, G. Distribuição e abundância do ictioplâncton entre Açú da Torre e Itapuã, Bahia, Brasil. In: CONGRESSO LATINOAMERICANO SOBRE CIÊNCIAS DEL MAR, 18, Trujillo, Anais... p.390-392. 1999.

MAFALDA Jr, P. (2000) Distribuição e abundância do ictioplâncton da Costa Norte da Bahia e suas relações com as condições oceanográficas. Tese Doutorado. FURG, Rio Grande, 165p.

MATSUURA, Y, SATO, G. Distribution and abundance of scombrid larvae in southern Brazilian waters. Bull. Mar. Sc., v. 31, n. 4, p. 824-832. 1981.

MATSUURA, Y. Distribution and abundance of skipjack (Katsuwonus pelamis) larvae in eastern Brazilian waters. Bolm. Inst. Oceanogr., S Paulo, v. 31, n. 2, p. 5-7. 1982.

MATSUURA, Y. Distribution and abundance of skipjack larvae off coasts of Brazil, Procc. ICCAT Conference on the Inter. Skipjack Year Program, 1-6. 1984.

McGOWEN, G. E. Coastal ichthyoplankton assemblages, with emphasis on the Southern California Bighth. Bull.Mar.Sci., v. 53, n. 2, p. 692-722. 1993.

MEDEIROS, C.; MACÊDO, S.; FEITOSA, F.; KOENING, M. L. Hydrography and phytoplankton biomass and abundance of north-east Brazilian waters. Archive of Fishery and Marine Research. v. 47, n. 2-3, p. 133-151. 1999.

MOSER, H. G.; RICHARDS, W. J.; COHEN, D. M.; FAHAY, M. P.; KENDALL Jr., A. W.; RICHARDSON, S. L. Ontogeny and systematics of fishes. Am. Soc. Ichthy. Herpert. Sp. Pub., 1984. 760p.

MOSER, H. G.; SMITH, P. E. Larval fish assemblage and oceanic boundaries. Bull. Mar. Sci., v. 53, n. 2, p. 283-289. 1993.

NASCIMENTO, E. D Ontogenia, distribuição e abundância da família Scombridae na Zona Econômica Exclusiva (ZEE) do Nordeste do Brasil. Recife, 2000.59p. Monografia, Departamento de Biologia, UFRPE.

NELSON, J. S. Fishes of world. New York, Jonh Wiley \& Sons. 1994.

NEUMANN-LEITÃO, S.; GUSMÃO, L. M.; SILVA, T.; NASCIMENTO-VIEIRA, D. A.; SILVA, A. P. Mesozooplankton biomass and diversity in coastal and oceanic waters off North-Eastern Brasil. Archive of Fishery and Marine Research. v. 47, n. 2-3, p. 153-165. 1999.

NEUMANN-LEITÃO, S. Impactos antrópicos na comunidade zooplanctônica estuarina. Porto de Suape - PE - Brasil. São Carlos. Recife, 1994, 273f. Tese (Doutorado) - Escola de Engenharia de São Carlos, Universidade de São Paulo, 
NISHIKAWA, Y.; RIMMER, D. Identification of larval tunas, billfishes and other Scombroid fishes (Suborder Scombroidei): an ilustrated guide. CSIRO Marine Laboratories Report, 186: 20p. 1987.

NONAKA, R. H.; MATSUURA, Y.; SUZUKI, K. Seazonal variation in larval fish assemblages in relation to oceanographic condition in the Abrolhos Bank region off eastern Brazil. Fish. Bull. n. 98, p. 767-784. 2000.

NORCROSS, B. L.; SHAW, R. F. Oceanic and estuarine transport of fish eggs and larvae: a review. Transactions of the American Fisheries Society, v. 113, p. 153-165. 1984.

OKUDA, T.; CAVALCANTI, L. B. Algumas condições oceanográficas na área nordeste de Natal. Trab. Inst. Oceanogr. da UFPE, Recife. v.3-4, n. 1, p. 27-31. 1963.

PAIVA, M. P. Recursos Pesqueiros Estuarinos e Marinhos do Brasil. Fortaleza, UFC Edições, 1997.286p.

PETERSON, R. G.; STRAMMA, L.Upper-level circulation in the south Atlantic ocean. Progress in Oceanography, v. 26, p. 1-75. 1991

RICHARDS, W. J. Distribution of tuna larvae (Pisces, Scombridae) in the northwestern gulf of Guinea and off Sierra Leone. Fis.Bull., v. 69, n. 3, p. 555-568. 1971

SILVA, V. R. F. Estudo sobre a comunidade ictioplanctônica da corrente do Brasil, entre Salvador,Ba e Aracaju, SE. Salvador, 1997, 60f. Monografia (Graduação) - Universidade Federal da Bahia.

SMITH, P. E.; RICHARDSON, S. L. Standard techniques for pelagic fish eggs and larvae surveys. FAO Fish. Tech. Pap., v. 175p. 1-100. 1977.

SOUZA P. M. M. Distribuição e abundância das larvas de peixe da família Scaridae, na Zona Econômica Exclusiva, entre Recife-PE e Salvador-BA, durante o REVIZEE Nordeste II. Salvador 1999, 22f.. Monografia, UFBA, Inst. De Biologia.

STRICKLAND, J. D. H.; PARSONS, T. R. A pratical handbook of seawater analysis. Bull. Fish. Res. Bd. Canada, v. 167, p. 1-311. 1972.

TRAVASSOS, P.; HAZIN, F. H. V.; ZAGAGLIA, J.; ADVÍNCULA, R.; SCHOBER, J. Thermohaline structure around seamounts and islands off North-Eastern Brazil. Archive of Fishery and Marine Research. v. 47, n. 2-3, p. 211-222. 1999.

VELAME, M. P. B. Composição, distribuição e abundância do ictioplâncton oceânico nos bancos oceânicos da Cadeia Norte e Arq. de Fernando de Noronha e de São Pedro e São Paulo. Salvador, 2001. Monografia (Bacharelado) - Instituto de Biologia Universidade Federal da Bahia.

ZAR, J. H. Bioestatistical Analysis. Prentice Hall, New York. 1984. 
